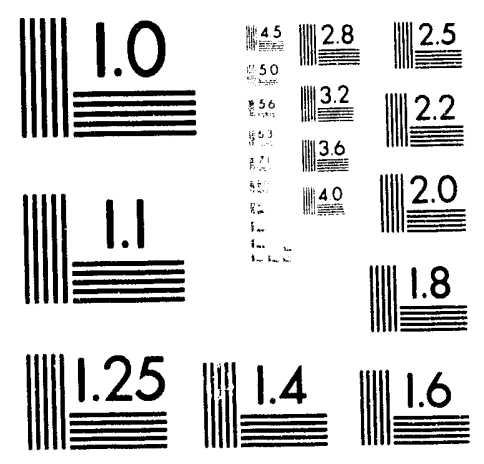



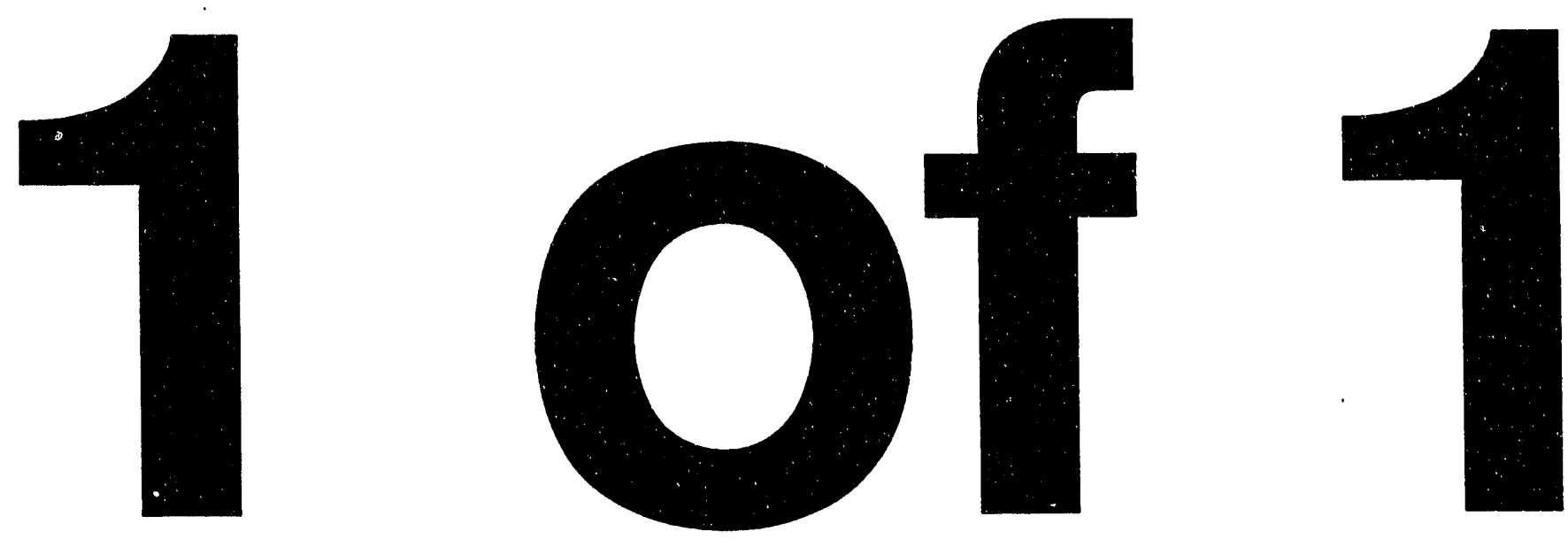


\title{
AN INTEGRO-ALGEURAIC EQUATION FOR HIGH FREQUENCY WAKE FIELDS IN A TUBE WITH SMOOTHLY VARYING RADIUS
}

\author{
Robert L. Warnock \\ Stanford Linear Accelerator Center \\ Stanford University, Stanford, CA 94309
}

\begin{abstract}
A technique to find the longitudinal wake field at frequencies above or below the tube cut-off is described. The round tube is infinite in length, and has an arbitrary, smooth variation of radius over a finite interval. A system of integro-algebraic equations enforces the boundary conditions cn the wall and the outgoing wave condition at infinity. The first step in an iterative solution of the system, valid for variations of tube radius with small derivative, yields a convenient formula for the impedance as a double integral. At low frequencies the formula gives Yokoya's result plus corrections that can be large. For high frequencies in the case of several wall undulations it gives a sequence of finite- $Q$ resonances. To avoid the limitations of the iterative method, a numerical solution of the system is carried out.
\end{abstract}

\section{DERIVATION OF THE EQUATION}

Other papers of this conference demonstrate the impressive utility of computer codes to determine electromagnetic fields in the presence of conducting walls of general form. Nevertheless. codes designed for arbitrary prohlems are not likely to answer all questions of interest. One instance is the wake field of a short or irregular bunch, with high-frequency components requiring an extremely fine mesh. The method described here may be a useful complement to general purpose programs. It will handle some problems that would normally require an overly fine mesh, it gives analytic formulas valid for a wide class of mild wall perturbations, and allows tests of accuracy merely by checking of boundary conditions. The method will be described for the case of the longitudinal wake field in a tube with circular cross section and infinite conductivity. It can be extended to treat transverse fields, wall resistance, and pipes of rectangular cross section.

We take cylindrical coordinates $(r, \phi, z)$ and suppose that the tube radius is given as $R(z)=$ $b-c s(z)$. where the function $s(z)$ is zero for $|z|>g$ : and not necessarily even in $z$. We assume that $s$ has a continuous first derivative $s^{\prime}$, normalized so that $\max \left|s^{\prime}\right|=1$; thus $s^{\prime}( \pm g)=0$. With this normalization, $f$ measures the effective strength of the wall perturbation; a perturbative method may succeed if $\epsilon$ is small compared to 1 .

We work in the frequency domain. with the time dependence exp(-iwt). Attention is restricted to positive values of $w$, which suffice to express the wake field, thanks to the reflection property of the impedance. $Z(\omega)=Z(-\omega)^{*}$. The source is assumed to be axisymmetric, a rigid bunch with total charge $q$ and charge density $\rho(r, \phi, z, t)=(q / 2 \pi) \lambda(z-\beta c t) f(r)$ where $\int \lambda(z) d z=1, \int f(r) r d r=1$. It follows that the only non-zero fields are $\left(E_{z}, E_{r}, H_{\phi}\right)$, all independent of $\phi$. All fields may be expressed in terms of $E_{z}$, which can be written as

$$
E_{z}(r, z, \omega)=\int_{\Gamma} d k e^{i k z} a(k, \omega) \frac{I_{o}(\chi r)}{I_{0}(\chi b)}+\epsilon_{z}(r, z, \omega),
$$

where $\lambda^{2}=k^{2}-(\omega / c)^{2}$, and $I_{0}$ is the modified Bessel function of the first kind. The Fourier transform $\dot{\epsilon}_{z}$ of the source term $\epsilon_{z}$ is any particular solution of the inhomogeneous radial wave

* Work supported by the Department of Energy, contract DE-AC03-76SF00515. 
equation for $E_{i}$. regular at $r=0$. A collvenient choice is

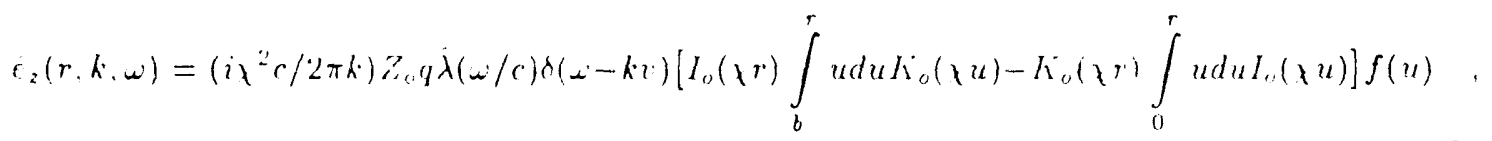

where $K_{0}$ is the modified Bessel function of the second kind, and $\lambda$ is the Fourier transform of $\lambda$. We work in m.k.s. units, with $Z_{o}=120 \pi \Omega$. The Fourier amplitude of the radial field is

$$
E_{r}(r, k, \omega)=-\frac{i k}{\lambda^{2}} \frac{\partial E_{z}(r, k, \omega)}{\partial r}
$$

The function $I_{0}\left({ }_{1} b\right)$ has simple zeros in the $k$-plane at the points $k= \pm k, s=1,2, \cdots$, where $k_{s}=\left((\omega b / c)^{2}-j_{o s}^{2}\right)^{1 / 2}$ is defined to be positive for $|\omega b / c|>j_{o s}$ and positive imaginary for $|w b / c|<j_{o s} ;$ the $j_{o}$ are the positive zeros of the Bessel function $J_{o}$. We define $s_{m}$ to be the number of real $\mathrm{k}_{\mathrm{s}}$. At any frequency above the !owest cutoff $\left(\omega b / c=j_{01}\right)$. there are $2 s_{n}$ zeros on the real axis. To dodge the corresponding real poles of the integrand in (1), the contour $\Gamma$ is indented slightly so as 10 go a bove the poles at $k=-k$, and below those at $k=k$. This choice enforces the outgoing wave boundary condition. We have assumed that $a(k, w)$ is analytic in $k$; our construction of solutions will in fact yield an entire fun ction of $k$.

The boundary condition on the wall is that $\mathbf{E}=\left(E_{r}, E_{z}\right)$ be perpendicular to the tangent vector $(d R, d z)$, or

$$
E_{z}(R(z), z, \omega)+R^{\prime}(z) E_{\Gamma}(R(z), z, \omega)=0
$$

This condition leads to an repation for $a(k, w)$ through the following steps: (i) write $E_{z}$ as in (1), and the corresponding expression for $E_{r}$ constructed from (3); (ii) take the Fourier transform of (4) with respert to $z$; (iii) subtract $I_{a}\left(x_{b}\right)$ from $I_{0}(x R(z))$ in the integrand. and notice that the compensating addition gives $\delta(k-l)$. The result is

$$
a(l, \omega)=\int_{i} d k \cdot M(l, k, \omega) a(k, \omega)+\mathcal{S}(l, \omega)
$$

where

$$
M(l, k, \omega)=\frac{1}{2 \pi} \int_{-g}^{g} d z \frac{e^{i(k-l \mid z}}{I_{0}\left(x^{b}\right)}\left[I_{o}\left(\chi^{b} b\right)-I_{0}(\chi R(z))+\frac{i k}{x} R^{\prime}(z) I_{o}^{\prime}(\chi R(z))\right]
$$

and $S$ is the Fourier transform of

$$
S(z, \omega)=-\int_{-\infty}^{\infty} d k \epsilon^{i k z}\left[e_{z}(R(z), z, \omega)-\frac{i k}{\lambda^{2}} R^{\prime}(z) \frac{\partial e_{z}}{\partial r}(R(z), z, \omega)\right] .
$$

Henceforth we treat only the relativistic limit. In that limit the source term simplifies: (2) and (7) yield

$$
S(z, \omega)=\frac{\epsilon Z_{0} s^{\prime}(z)}{2 \pi R(z)} \lambda(\omega / c) e^{i \omega z / c}
$$

Below cutoff, (5) is an integral equation [1] for $a(k, \omega)$. Above cutoff it is an integroalgebraic equation, since the values $a(k, \omega)$ at the poles on the real axis constitute a discrete set of unknowns to be determined along with the continuous, nonpolar part. These values determine the amplitudes of outgoing waves. 
form

By reversing the order of integrals we see that any solution of (5) may he written in the

$$
a(k, w)=\frac{1}{2 \pi} \int_{-g}^{g} \epsilon^{-i k_{i}} \Phi(z, \omega) d z
$$

Since the integral is finite, $a(k, \omega)$ is an entire function of $k$, as promised.

\section{FORMULAS FOR THE IMPEDANCE, FROM THE LOWEST ITERATE}

An integration by parts on the first two terms of $(6)$ puts the kernel in the form

$$
M(l, k, \omega)=\frac{c}{2 \pi} \frac{k l-(\omega / c)^{2}}{\lambda(k-l)} \int_{-g}^{g} d z e^{.(k-l) z} R^{\prime}(z) \frac{I_{o}^{\prime}(\chi R(z))}{I_{o}(\chi b)} .
$$

This shows that the kernel is formally $\mathcal{O}(\epsilon)$, and therefore suggesis that the equation (5) might be solved by iteration when $\epsilon$ is small. The first approximation is obtained by putting $a=\hat{S}$ under the integral in $(5)$. Since the impedance is proportional to $a(\omega / c, \omega)$, and $\hat{\mathcal{S}}(\omega / c, \omega)=0$, the lowest order impedance is $\mathcal{O}\left(c^{2}\right)$.

To evaluate the approximated integral of $(5)$ at the synchronous point $k=\omega / c$ we express $\mathcal{S}$ in terms of its Fourier transform and reverse integration order to obtain

$$
a(\omega / c, \omega) \approx \frac{1}{2 \pi} \int_{-g}^{g} d z \epsilon^{-i \omega^{\prime} z / c} R^{\prime}(z) \int_{-g}^{g} d z^{\prime} \mathcal{S}\left(z^{\prime}, \omega\right) K\left(z, z^{\prime}, \omega\right),
$$

where

$$
h\left(z, z^{\prime}, w\right)=\frac{1}{2 \pi i} \frac{\omega}{c} \int_{\Gamma} d k \epsilon^{i k\left(z-z^{\prime}\right)} \frac{I_{o}^{\prime}(\lambda R(z))}{\lambda I_{o}(\chi b)}
$$

The integral (12) converges exponentially if $R<b$, but diverges for $R>b$. The divergence is an unwanted limitation since we wish to allow arbitrary $R$. By performing a contour distortion one can continue the integral analytically from $R<b$ to $R>b$, and incidentally gain other benefits. Taking $R<b$ we let the contour become an infinite semi-circle in the upper (lower) half-plane for $z-z^{\prime}$ positive (negative). The result is

$$
K\left(z, z^{\prime}, \omega\right)=\frac{\omega}{c} \sum_{s=1}^{\infty} \frac{J_{1}\left(j_{o s} R(z) / b\right)}{k_{s}(\omega) b J_{1}\left(j_{o s}\right)} \epsilon^{\left.i k_{s}(\omega) \mid z-z^{\prime}\right)} .
$$

For $z \neq z^{\prime}$ the sums converge exponentially, regardless of the value of $R$. At $z=z^{\prime}$ and $R=b$ the sum diverges, but if the integral on $z^{\prime}$ is performed first there will be an extra inverse power of $k$, and quadratic convergence, uniform in $R$.

The formula (11) now involves powers of $\epsilon$ higher than the second through the presence of $R(z)$ in the denominator of $(8)$ and in (13). To pick out just the $\epsilon^{2}$ part we put $R(z)=b$ in both locations. Invoking the usual definition of the impedance in terms of the wake potential, we find $Z(\omega)=-2 \pi a(\omega / c, \omega) /(q \dot{\lambda}(\omega / c))$. Then from (8), (11), and (13) we have the impedance to lowest order in $\epsilon$ as

$$
Z(\omega)=\frac{\omega Z_{o} \epsilon^{2}}{2 \pi c b^{2}} \sum_{s=1}^{\infty} \frac{1}{k_{s}(\omega)} \int_{-g}^{g} d z \int_{-g}^{g} d u s^{\prime}(z) s^{\prime}(u) \epsilon^{i k_{a}(\omega)|z-u|-i \omega(z-u) / c} .
$$

Below cutoff the $k$, are all positive imaginary, and the impedance is reactive as required; (the integral is real, since the integrand goes into its complex conjugate on $z \rightarrow u$ ). 
A closer look shows that the formula (14) is actually invalid for the frequency $w$ in a small neighborhood of each traveling wave cutoff, where wb/r $=j_{0 s}$. The kernel $K$ has an inverse square-root singularity at such points, owing to the factor $1 / k$, in (13). It is therefore not small near such frequencies, and the iterative method fails. The same singularity appears in the field expansion (1). from the residue of the pole as it strikes the real axis. It is cancelled by a corresponding zero of $a(k, \omega)$ at $k= \pm k$, so that the amplitude of the newly appearing outgoing wave is finite. We have verified that this mechanism operates in the numerical calculation of the following section, but it is a "nonperturbative" effect that cannot take place in a lowest order calculation. In plotting results from (14), we delete small neighborhoods of the bad points, and let the plotting program interpolate nearby values to fill in the gaps. This is justified by the smooth behavior of $Z$ found in the mumerical solutions

As a first application of (14) we take the frequency below cutoff and perform a partial integration of the $u$-integral. For this we assume that $s^{\prime \prime}$ is at least piecewise continuous. The integrated term yields an s-sum that can be cvaluated (namely $\sum, j_{o s}^{-2}=1 / 4$ ), and the corresponding contribution to the impedance is the result obtained previously by Yokoya [2] through a much more involved method:

$$
Z(\omega)=-i \frac{\omega}{4 \pi r} Z_{0} c^{2} \int_{-g}^{g} d z\left[s^{\prime}(z)\right]^{2}
$$

The term remaining after partial integration can be large, however, and is nonlinear in $\omega$. One expects some correction in any case, since the fields in general must depend on $b / g$, while there is no such dependence in (15). As an example we take $s(z)=d(1+\cos (\pi z / g)) / 2, \quad|z|<g$. for which $t=\pi|d| /(2 g)$. In the small $a$ limit a full evaluation of $(14)$ gives in this case

$$
Z(\omega)=-i \frac{\omega g}{r} Z_{o} \frac{\varepsilon^{2}}{4 \pi}\left[1+\left(1-2 \frac{I_{1}(\pi b / g)}{(\pi b / g) I_{o}(\pi b / g)}\right)+4 \pi^{2}\left(\frac{b}{g}\right)^{3} \sum_{s=1}^{\infty} \frac{1-e^{-2 j_{0 . g} / b}}{j_{o s}\left(j_{o s}^{2}+(\pi b / g)^{2}\right)^{2}}\right]
$$

The first term in the square bracket is the Yokoy term, the second term vanishes as $(b / g)^{2}$ and the third as $(b / g)^{3}$, when $g \rightarrow x$. The correction to the Yokoya term (mainly from the second term) is ahout $50 \%$ at $2 g / b=2$, and approaches $100 \%$ as $g \rightarrow 0$. Figure 1 shows the sum of the second and third terms as a function of $2 \mathrm{~g} / \mathrm{b}$.

As an example for arbitrary frequency, we generalize the previous example to allow many oscillations of the radius, taking $s(z)=d(1+\cos (\pi p z / g)) / 2$, where $p$ is an odd integer, thus $\epsilon=\pi p|d| /(2 g)$. An exart evaluation of (14) for this model yields

$$
Z(\omega)=\frac{\omega b}{c} Z_{o} \frac{\epsilon^{2}}{2 \pi}\left[-i \frac{g}{2 b}[h(\omega)+h(-\omega)]+\left(\frac{\pi p b}{g}\right)^{2} \sum_{s=1}^{\infty} \frac{1}{b k_{s}(\omega)}\left[f\left(k_{s}(\omega), \omega\right)+f\left(k_{s}(\omega),-\omega\right)\right]\right]
$$

where

$$
h(\omega)=\frac{I_{1}(\xi)}{\xi I_{0}(\xi)} \quad, \quad \xi=\left[\left(\frac{\pi p b}{g}\right)^{2}+2 \frac{\omega b}{c}\left(\frac{\pi p b}{g}\right)\right]^{1 / 2} \quad, \quad f(k, \omega)=\frac{1-\exp [2 i(k-\omega / c) g]}{b^{4}\left[(k-\omega / c)^{2}-(\pi p / g)^{2}\right]^{2}}
$$

The first term is imaginary at all $\omega$, and so is the sum for $s>s_{m}$. The sum for $s \leq s_{m}$ is complex, and of course present only above cutoff. Its real part is nonnegative as it should be, corresponding to energy lost by the bunch to outgoing waves. The term $h(-\omega)$ has poles, but they are cancelled by corresponding poles in the sum on $s$.

Figure 2 shows a plot of formula (17) for $p=1, b=1 \mathrm{~cm}, g=6 \mathrm{~cm}, d=0.3 \mathrm{~cm}$, thus $\epsilon=0.078$. When the number of wall undulations is increased, a sequence of resonances of decreasing $Q$ appears. Figures 3 and 4 show results for $p=5, b=1 \mathrm{~cm}, g=6 \mathrm{~cm}, d=0.06 \mathrm{~cm}$, thus the same $\epsilon=0.078$. Since the impedance simply scales with $d^{2}$, it retains its complicated form for arbitrarily small $d$, while decreasing in magnitude quadratically. 
Correction to Yokoya Formula

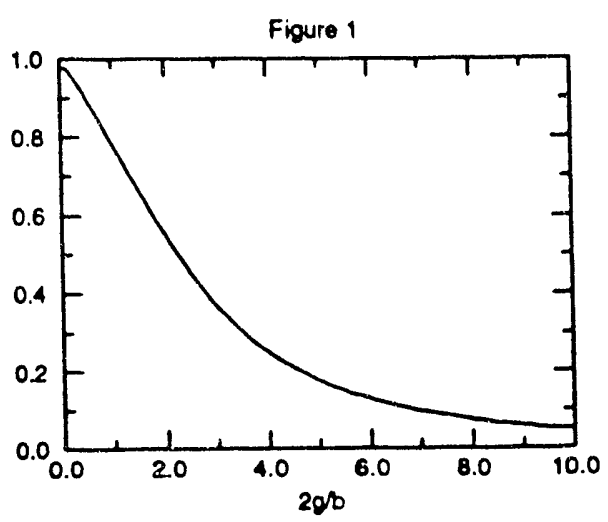

$b=1 \mathrm{~cm} g=6 \mathrm{~cm} \quad d=0.06 \mathrm{~cm} \quad p=5$

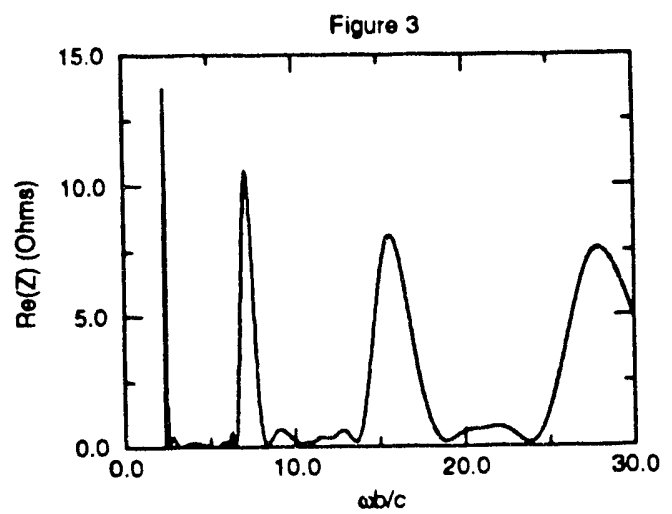

$b=1 \mathrm{~cm} \quad g=6 \mathrm{~cm} \quad d=0.3 \mathrm{~cm} \quad p=1$

Figure 2

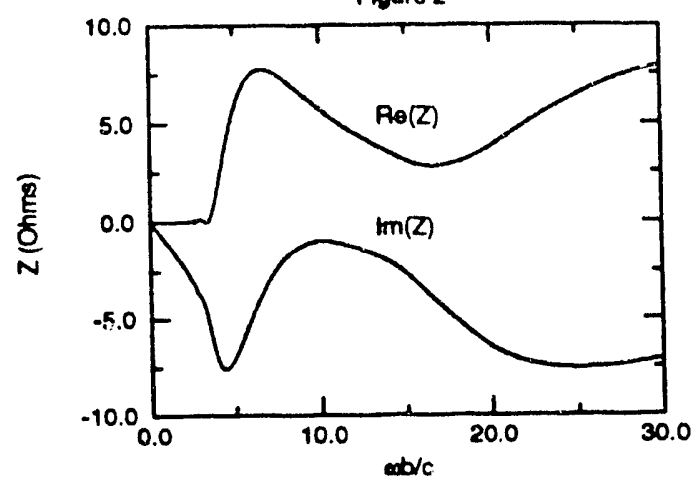

$b=1 \mathrm{~cm} \quad g=6 \mathrm{~cm} \quad d=0.06 \mathrm{~cm} \quad p=5$

Figure 4

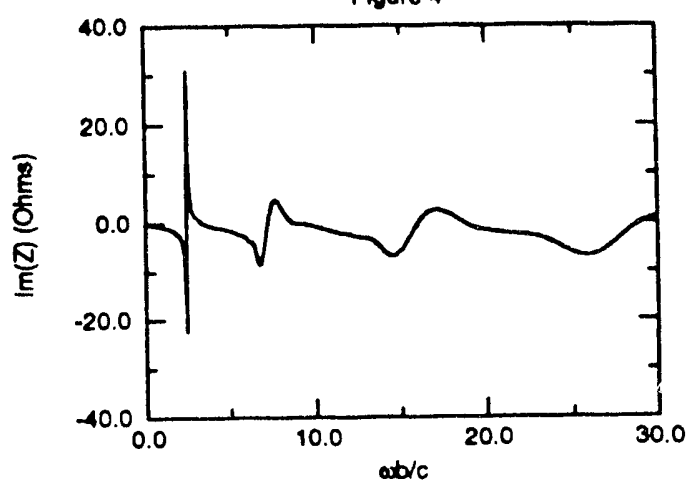




\section{NUMERICAL SOLUTION}

For a numerical solution we formulate the integral equation in a different way. In (1) we translate the contour a finate distance a into the upper (lower) half-plane for $z>0(z<0)$. respertively. Thus we have two separate representations of $E_{z}$ for the two signs of $z$, as follows:

$$
\begin{aligned}
E_{z}(r, z, \omega)-\epsilon_{z}(r, z, \omega)= & \epsilon^{-\alpha|z|} \int_{-\infty}^{\infty} d v\left[\frac{a(k, \omega) I_{0}(\chi r)}{I_{o}(\chi b)}\right]_{k=v \pm i \alpha} e^{i v z} \\
& \pm 2 \pi i \sum_{s=1}^{n} \frac{j_{0 s} J_{o}\left(j_{0,} r / b\right)}{b J_{1}\left(j_{0 s}\right)}\left[\frac{a(k, \omega) \epsilon^{i k z}}{k b}\right]_{k= \pm k_{o}(\omega)}
\end{aligned}
$$

The second term represents outgoing waves moving to the right (left), plus any evanescent waves with $0<\left|\operatorname{Im} k_{s}\right|<\alpha$. The integral decays exponentially as $|z| \rightarrow \infty$. Each traveling or evanescent wave in the second term of (19) automatically satisfies the boundary condition $E_{z}(b, z, \omega)=0$ in the region $|z|>g$ where the tube has constant radius $b$. Moreover, in some region $|z|>h>g$ beyond the wall perturbation the decaying integral will $b \circ$ negligible if $\alpha$ is sufficiently large. Thus to satisfy the wall boundary condition for the entire infinite tube we need only take steps to impose it on the finite region $|z|<h$. That can be done numerically by taking a discrete Fourier transform of the boundary condition over this region.

Let us first derive the exact continum equations, and later discretize. We substitute the composite representation (19) of $E_{z}$ and the corresponding form for $E_{r}$ in the boundary condition (4), subtract and add $I_{0}\left(x^{b}\right)$ as we did before, then move the contour of the added term (which entails no poles) back to the real axis. Then take the Fourier transform on $z$, and get a freestanding $a(l, \omega)$ on the left hand side. Finally continue the result analytically to $l=u \pm i \alpha$ and $l= \pm k_{t}$ to obtain the following system of integro-algebraic equations:

$$
\begin{aligned}
a(u \pm i \alpha) & =\int_{-\infty}^{\infty} d v\left[K_{+}(u \pm i \alpha, v+i \alpha) a(v+i \alpha)+K_{-}(u \pm i \alpha, v-i \alpha) a(v-i \alpha)\right] \\
& +\sum_{s=1}^{n}\left[L_{+}\left(u \pm i \alpha, k_{s}\right) a\left(k_{s}\right)+L_{-}\left(u \pm i \alpha,-k_{s}\right) a\left(-k_{s}\right)\right] \\
& +\dot{\mathcal{S}}(u \pm i \alpha), \quad u \in[-\infty, \infty], \\
a\left( \pm k_{t}\right) & =\int_{-\infty}^{\infty} d v\left[K_{+}\left( \pm k_{t}, v^{\prime}+i \alpha\right) a(v+i \alpha)+K_{-}\left( \pm k_{t}, v-i \alpha\right) a(v-i \alpha)\right] \\
& +\sum_{s=1}^{n}\left[L_{+}\left( \pm k_{t}, k_{s}\right) a\left(k_{s}\right)+L_{-}\left( \pm k_{t},-k_{s}\right) a\left(-k_{s}\right)\right] \\
& +\mathcal{S}\left( \pm k_{t}\right), \quad t=1,2, \cdots, n .
\end{aligned}
$$

We have suppressed all reference to $\omega$, and have defined the kernel functions as

$$
\begin{aligned}
& K_{ \pm}(l, k)=\frac{ \pm 1}{2 \pi} \int_{0}^{ \pm g} d z e^{i(k-l) z} \frac{1}{I_{o}(\chi b)}\left[I_{o}(\chi b)-I_{o}(\chi R(z))+\frac{i k}{\chi} R^{\prime}(z) I_{o}^{\prime}(\chi R(z))\right], \\
& L_{ \pm}\left(l, k_{s}\right)= \pm i \int_{0}^{ \pm g} d z e^{i(k,-l) z} \frac{j_{o s}}{k_{s} b^{2} J_{1}\left(j_{o s}\right)}\left[-J_{o}\left(j_{o s} R(z) / b\right)+\frac{i k_{s} b}{j_{o s}} R^{\prime}(z) J_{1}\left(j_{o s} R(z) / b\right)\right]
\end{aligned}
$$

In the numerical realization we first redefine the integration variable $v$ by a translation so that its origin corresponds to the point $k=\omega / c$, since the source term is small outside a 
neightorhood of that point. We then write each t-integral of (19) as a discrete Fourier transform on a finite interval $\left[-l^{\prime}, l^{\circ}\right]$. The inverse discrete transform to enforce the boundary condition is defined for $z \in[-h, h]$. The choice of the parameters $h, V, \alpha$ requires a little experimentation. but is not very critical. The contour displacement a must be chosen so that the contour is not too close to an imaginary $k_{s}$. In examples studied to date, values of $\alpha g$ in the range 3 to 10 have been satisfactory. All experimentation with solution control parameters is guided by checking satisfaction of the wall boundary condition. Since the fields we construct are automatically solutions of Maxwell's equations, and satisfy the outgoing wave condition, this is a definitive test of accuracy of the solution.

Since we do numerical Fourier anuysis of functions with a factor $s^{\prime}(z)$, the method works best if $s^{\prime \prime}$ is continuous. Accordingly, we give results for a model with such continuity, $s(z)=$ $(d / 4)(1+\cos (\pi j ; / g))^{2},|z|<g$, with $p$ odd

Figure 5 shows the impedance computed for $p=1, b=1 \mathrm{~cm}, g=6 \mathrm{~cm}, d=0.3 \mathrm{~cm}$, up to a high enough frequency $(134 \mathrm{GHz})$ to compute the wake field of a $1.2 \mathrm{~mm}$ Gaussian bunch. Note the similarity to Figure 2, recalling that $s(z)$ for Figure 2 is the square root of the present $s(z)$. The computation used 192 conplex Fourier amplitudes to represent the two discretized integrals. The computation time was 4 to 5.5 seconds per frequency on the IBN 3090; the larger times occur when there are several outgoing waves. Figure 6 shows the corresponding wake voltage at a distance $x$ from the center of the bunch, in units of bunch length $\sigma=1.2 \mathrm{~mm}$. Figure $i$ shows a test of accuracy for this solution, namely a plot of $\eta=\left|E_{t}(R(z), z, \omega) / E_{n}(R(z), z, \omega)\right|$ at $\omega b / c=4.8$ where $E_{t}$ and $E_{n}$ are the tangential and normal components of $\mathbf{E}$ on the wall. The boundary condition is well satisfied at this frequency, with $\eta<10^{-6}$; the accuracy degrades somewhat at higher frequencies but overall $\eta<10^{-4}$. Figure 8 derives from the field on axis; it is a plot of $\operatorname{Re}\left[E_{z}(r=0, z \cdot \omega) \exp \left(i_{\omega} z / c\right)\right]$ at $\omega b / c=14.4$. The oscillations at positive : correspond to the superposition of several outgoing waves with incommensurate wavelengths. The bunch and these waves move to the right: left-going waves are present but very weak, and become strong only in certain narrow frequency bands. Figure 9 shows part of the solution of the equations (20), the continuum component $\operatorname{Re}\left[a(k, \omega) / I_{o}(\chi b)\right]$ for $k=v+i \alpha$ on the upper contour, at $\omega b / c=14.4$. Figure 10 shows an axial field plot like that of Figure 8 but for $p=5$ (with $d$ reduced by a factor of 5 to give the same $c$ ), at $\omega b / c=4.8$.

\section{CONCLUSION}

We have shown that formula (14) predicts a rich pattern of high frequency effects. A detailed study of this formula, which is easy to evaluate for many different forms of the wall perturbation, should lead to useful insights regarding parameter dependence of wake fields. We have shown that the numerical treatment of the integro-algebraic system can produce very accurate solutions of the boundary value problem. Comparison and parallel further development of the analytic and numerical approaches should be rewarding.

I wish to thank Robert Gluckstern, Karl Bane, and J. Scott Berg for much good advice and technical help.

\section{REFERENCES}

1. An equation with the same kernel was derived by S. S. Kurennoy and S. V. Purtov, Particle Accelerators 36223 (1992). Their form of the source term is incorrect, however, as is their treatment of frequencies above cutoff.

2. K. Yokoya, CERN SL/90-88 (AP) (1990). 
$b=1 \mathrm{~cm} \quad g=6 \mathrm{~cm} \quad d=0.3 \mathrm{~cm} \quad p=1$

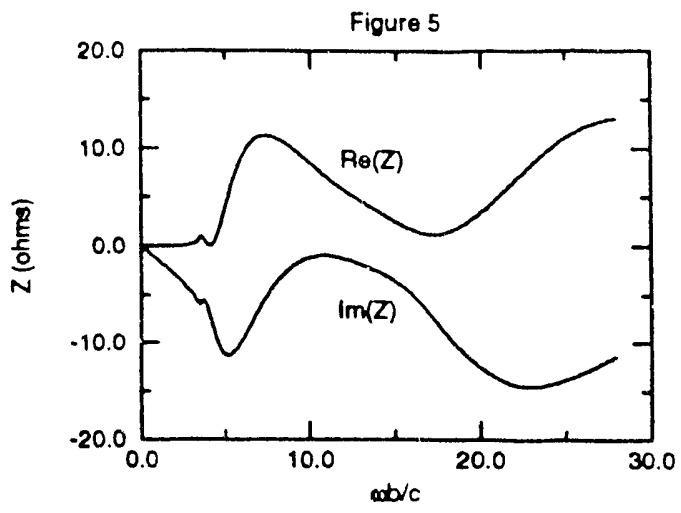

$\left|E_{1}(R(z), z, \omega) / E_{n}(R(z), z, \omega)\right|$

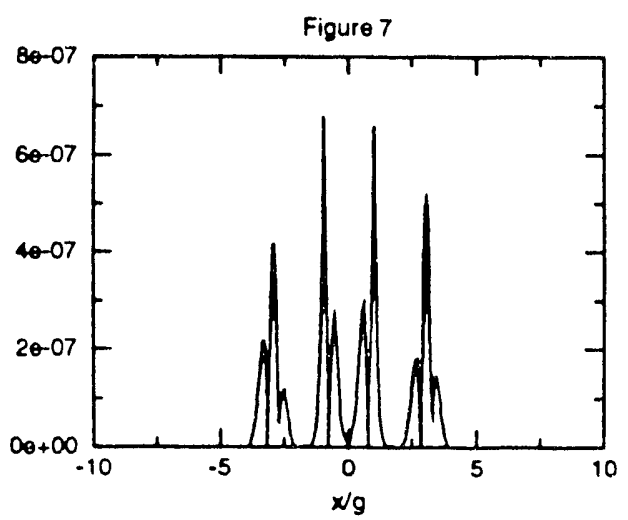

$\operatorname{Re}\left(a(k, \omega) / I_{0}(\chi b)\right) \quad k=v+i \alpha \quad \omega b / c=14.4$

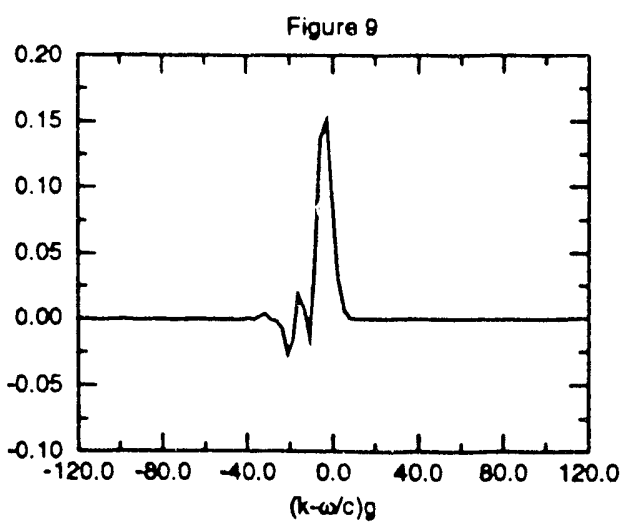

Wake voltage $\sigma=1.2 \mathrm{~mm}$

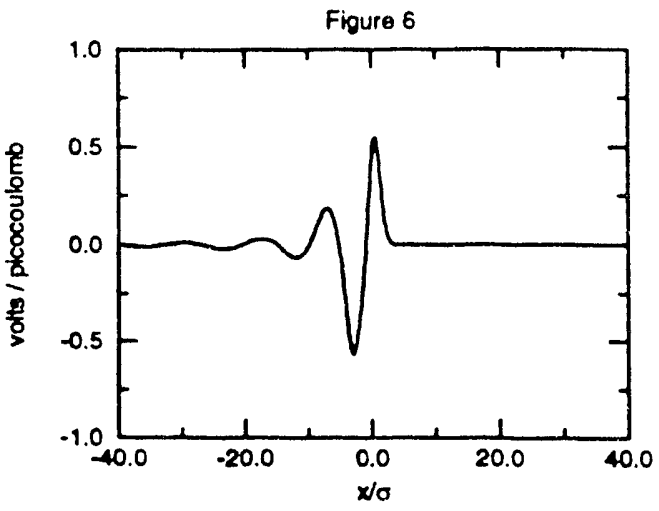

$\operatorname{Re}\left(E_{2}(0, z, \omega) e^{\log z / c}\right) \quad \omega b / c=14.4$

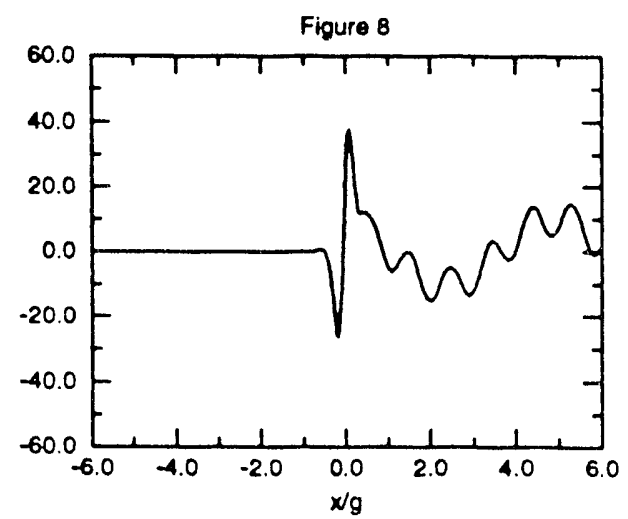

$\operatorname{Re}\left(E_{z}(0, z, \omega) e^{i \operatorname{koz} / c}\right) p=5 \omega b / c=4.8$

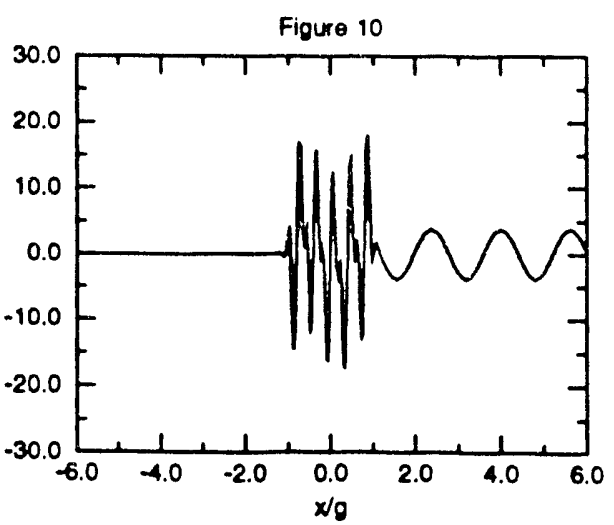



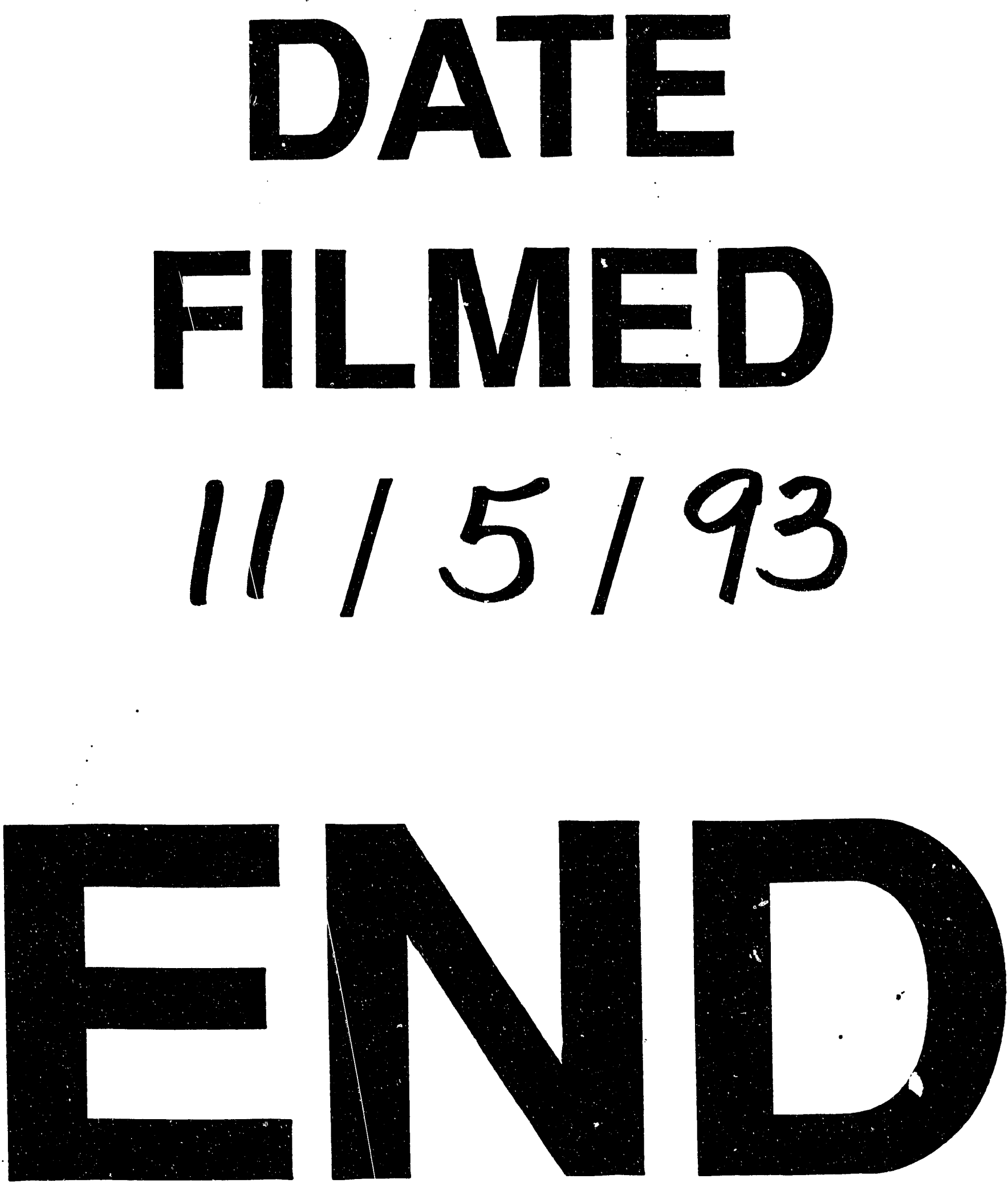
